\title{
High-contrast Polarimetry Observation of the T Tau Circumstellar Environment
}

Yi Yang ${ }^{1,2}$ (1) , Satoshi Mayama ${ }^{3}$ (i) , Saeko S. Hayashi ${ }^{1,2}$, Jun Hashimoto ${ }^{4}$, Roman Rafikov $^{5,6}$, Eiji Akiyama ${ }^{7}$ (1), Thayne Currie ${ }^{8}$ (1), Markus Janson $^{9}$ (1), Munetake Momose ${ }^{10}$ (1), Takao Nakagawa ${ }^{11}$ (1), Daehyeon $\mathrm{Oh}^{1,2,12}$ (1), Tomoyuki Kudo ${ }^{8}$ (1), Nobuhiko Kusakabe $^{4}$, Lyu Abe ${ }^{13}$, Wolfgang Brandner ${ }^{14}$, Timothy D. Brandt ${ }^{15}$ (D) , Joseph C. Carson ${ }^{16}$, Sebastian Egner ${ }^{17}$, Markus Feldt $^{14}$, Miwa Goto ${ }^{18}$, Carol A. Grady ${ }^{19,20,21}$, Olivier Guyon ${ }^{4,8,22}$ (1), Yutaka Hayano ${ }^{1,2,8}$ (1], Masahiko Hayashi ${ }^{1,2}$, Thomas Henning $^{14}$, Klaus W. Hodapp ${ }^{23}$ (D) , Miki Ishii $^{2}$, Masanori Iye ${ }^{2}$, Ryo Kandori ${ }^{2}$ (iD, Gillian R. Knapp ${ }^{24}$ (D), Jungmi Kwon ${ }^{11}$ (D),

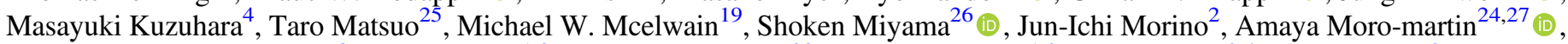
Tetsuo Nishimura ${ }^{8}$, Tae-Soo Pyo ${ }^{1,8}$ (1) , Eugene Serabyn ${ }^{28}$, Takuya Suenaga ${ }^{1,2}$, Hiroshi Suto ${ }^{2,4}$, Ryuji Suzuki ${ }^{2}$, Yasuhiro H. Takahashi ${ }^{2,29}$, Michihiro Takami ${ }^{30}$ (1) , Naruhisa Takato ${ }^{1,8}$, Hiroshi Terada ${ }^{2}$ (1) , Christian Thalmann $^{31}$ (1), Edwin L. Turner ${ }^{24,32}$, Makoto Watanabe ${ }^{33}$ (10) John Wisniewski ${ }^{34}$ (D) , Toru Yamada $^{35}$, Hideki Takami ${ }^{1,2}$, Tomonori Usuda ${ }^{1,2}$ (1) , and Motohide Tamura ${ }^{2,4,29}$ (1)

${ }^{1}$ Department of Astronomical Science, SOKENDAI (The Graduate University for Advanced Studies), 2-21-1 Osawa, Mitaka, Tokyo 181-8588, Japan; yi.yang @ nao.ac.jp

${ }^{2}$ National Astronomical Observatory of Japan (NAOJ), National Institutes of Natural Sciences (NINS), 2-21-1 Osawa, Mitaka, Tokyo 181-8588, Japan

${ }^{3}$ SOKENDAI(The Graduate University for Advanced Studies), Shonan International Village, Hayama-cho, Miura-gun, Kanagawa 240-0193, Japan ${ }^{4}$ Astrobiology Center, NINS, 2-21-1, Osawa, Mitaka, Tokyo 181-8588, Japan

${ }^{5}$ Astrophysics Department, Institute for Advanced Study, Princeton, NJ 08540, USA

${ }^{6}$ Department of Applied Mathematics and Theoretical Physics, Centre for Mathematical Sciences, University of Cambridge, Wilberforce Road, Cambridge CB3 0WA, UK

${ }^{7}$ Institute for the Advancement of Higher Education, Hokkaido University, Kita 17, Nishi 8, Kita-ku, Sapporo, Hokkaido, 060-0817, Japan ${ }^{8}$ Subaru Telescope, NAOJ, NINS, 650 North A'ohoku Place, Hilo, HI 96720, USA

${ }^{9}$ Department of Astronomy, Stockholm University, AlbaNova University Center, SE-106 91 Stockholm, Sweden

${ }^{10}$ College of Science and Center for Astronomy, Ibaraki University, 2-1-1 Bunkyo, Mito, 310-8512, Japan

${ }^{11}$ Institute of Space and Astronautical Science, Japan Aerospace Exploration Agency, 3-1-1 Yoshinodai, Chuo-ku, Sagamihara, Kanagawa 252-5210, Japan

${ }_{12}$ National Meteorological Satellite Center, 64-18 Guam-gil, Gwanghyewon-myeon, Jincheon-gun, Chungbuk, Republic of Korea

${ }^{13}$ Laboratoire Lagrange (UMR 7293), Universite de Nice-Sophia Antipolis, CNRS, Observatoire de la Coted'azur, 28 Avenue Valrose, F-06108 Nice Cedex 2, France ${ }^{14}$ Max Planck Institute for Astronomy, Königstuhl 17, D-69117 Heidelberg, Germany

${ }^{15}$ Department of Physics, Broida Hall, University of California, Santa Barbara, CA 93106-9530, USA

${ }^{16}$ Department of Physics and Astronomy, College of Charleston, 58 Coming Street, Charleston, SC 29424, USA

${ }^{17}$ European Southern Observatory, Karl Schwarzschildstr. 2, Garching D-85748, Germany

${ }^{18}$ Universitäts-Sternwarte München, Ludwig-Maximilians-Universität, Scheinerstr. 1, D-81679 München, Germany

${ }^{19}$ Exoplanets and Stellar Astrophysics Laboratory, Code 667, Goddard Space Flight Center, Greenbelt, MD 20771, USA ${ }^{20}$ Eureka Scientific, 2452 Delmer, Suite 100, Oakland, CA 96002, USA

${ }^{21}$ Goddard Center for Astrobiology, NASA Goddard Space Flight Center, Greenbelt, MD 20771, USA

${ }^{22}$ Steward Observatory, University of Arizona, 933 N Cherry Avenue, Tucson, AZ 85719, USA

${ }^{23}$ Institute for Astronomy, University of Hawaii, 640 N. A'ohoku Place, Hilo, HI 96720, USA

${ }^{24}$ Department of Astrophysical Science, Princeton University, Peyton Hall, Ivy Lane, Princeton, NJ 08544, USA

${ }^{25}$ Department of Earth and Space Science, Graduate School of Science, Osaka University, 1-1 Machikaneyamacho, Toyonaka, Osaka, 560-0043, Japan

${ }_{26}$ Hiroshima University, 1-3-2 Kagamiyama, Higashihiroshima, Hiroshima, 739-8511, Japan

${ }^{27}$ Department of Astrophysics, CAB-CSIC/INTA, E-28850 Torrejón de Ardoz, Madrid, Spain

${ }^{28}$ Jet Propulsion Laboratory, California Institute of Technology, M/S 171-113 4800 Oak Grove Drive Pasadena, CA 91109, USA

${ }_{30}^{29}$ Department of Astronomy, The University of Tokyo, 7-3-1 Hongo, Bunkyo-ku, Tokyo 113-0033, Japan

${ }^{30}$ Institute of Astronomy and Astrophysics, Academia Sinica, P.O. Box 23-141, Taipei 10617, Taiwan

${ }^{31}$ Swiss Federal Institute of Technology (ETH Zurich), Institute for Astronomy, Wolfgang-Pauli-Strasse 27, CH-8093 Zurich, Switzerland

${ }^{32}$ Kavli Institute for Physics and Mathematics of the Universe, The University of Tokyo, 5-1-5 Kashiwanoha, Kashiwa, Chiba 277-8568, Japan

${ }_{33}$ Department of Cosmosciences, Hokkaido University, Kita-ku, Sapporo, Hokkaido 060-0810, Japan

${ }^{34}$ H. L. Dodge Department of Physics \& Astronomy, University of Oklahoma, 440 W Brooks Street, Norman, OK 73019, USA

${ }^{35}$ Astronomical Institute, Tohoku University, Aoba-ku, Sendai, Miyagi 980-8578, Japan

Received 2017 November 29; revised 2018 May 16; accepted 2018 May 18; published 2018 July 12

\begin{abstract}
We conducted high-contrast polarimetry observations of T Tau in the $H$-band, using the High Contrast Instrument for the Subaru Next Generation Adaptive Optics instrument mounted on the Subaru Telescope, revealing structures as near as 0 ". 1 from the stars T Tau N and T Tau S. The whole T Tau system is found to be surrounded by nebulalike envelopes, and several outflow-related structures are detected in these envelopes. We analyzed the detailed polarization patterns of the circumstellar structures near each component of this triple young star system and determined constraints on the circumstellar disks and outflow structures. We suggest that the nearly face-on circumstellar disk of T Tau $\mathrm{N}$ is no larger than $0 . " 8$, or $117 \mathrm{au}$, in the northwest, based on the existence of a hole in this direction, and no larger than 0 "! 27 , or $40 \mathrm{au}$, in the south. A new structure, "N5," extends to about 0 ". 42 , or $59 \mathrm{au}$, southwest of the star, and is believed to be part of the disk. We suggest that T Tau S is surrounded by a highly inclined circumbinary disk with a radius of about $0 . " 3$, or $44 \mathrm{au}$, with a position angle of about $30^{\circ}$, that is misaligned with the orbit of the T Tau S binary. After analyzing the positions and polarization vector patterns of the outflow-related structures, we suggest that T Tau S should trigger the well-known E-W outflow, and is also likely to be responsible for a southwest precessing outflow "coil" and a possible south outflow.
\end{abstract}


Key words: protoplanetary disks - binaries: general (T Tau) - stars: pre-main sequence - stars: variables: T Tauri, Herbig Ae/Be

\section{Introduction}

As a prototype of the large class of $\mathrm{T}$ Tauri pre-main sequence stars, $\mathrm{T}$ Tau has attracted considerable attention from astronomers studying star and planet formation processes. $\mathrm{T}$ Tau is located about $146.7 \pm 0.6 \mathrm{pc}$ from us (Loinard et al. 2007) and has an age of about 1-2 Myr (Kenyon \& Hartmann 1995). It is actually a triple system, consisting of the north single star $\mathrm{T}$ Tau $\mathrm{N}$ and the south binary $\mathrm{T}$ Tau Sa/Sb. T Tau N, which has a mass of about $1.95 M_{\odot}$ (Köhler et al. 2016), is believed to be a class II young stellar object (YSO) (Furlan et al. 2006; Luhman et al. 2010). T Tau S, located about 0 "! 7 south of $\mathrm{T}$ Tau $\mathrm{N}$, was first discovered by Dyck et al. (1982) and could be a class I YSO (Furlan et al. 2006; Luhman et al. 2010). Koresko (2000) discovered that $\mathrm{T}$ Tau $\mathrm{S}$ is actually a binary $\mathrm{Sa} / \mathrm{Sb}$ with a separation of about 0 "' 1 , with masses of $2.12 \pm 0.10 M_{\odot}$ for $\mathrm{Sa}$ and $0.53 \pm 0.06 M_{\odot}$ for Sb (Köhler et al. 2016). The orbit of T Tau $\mathrm{Sa} / \mathrm{Sb}$ has a semimajor axis of $12.5_{-0.3}^{+0.6}$ au and an eccentricity of $0.56_{-0.09}^{+0.07}$. The orbit of the T Tau N-S system is not well constrained, but is likely to have a semimajor axis of $430_{-250}^{+790}$ au and an eccentricity of $0.7_{-0.4}^{+0.2}$ (Köhler et al. 2016).

Although T Tau has been studied extensively, there are still some important issues that need to be resolved for this system. The first issue is the circumstellar disk structures in the system. Akeson et al. (1998) made $3 \mathrm{~mm}$ continuum observations of $\mathrm{T}$ Tau $\mathrm{N}$ and estimated that it might have a nearly face-on disk with an outer radius of about $41 \mathrm{au}$, but their beam-size $(0$. $59 \times 0$ ". 39$)$ was not sufficient to reveal the details. Other research has given quite different results, such as Gustafsson et al. (2008), who estimated the outer radius as 85-100 au, corresponding to about 0 ." $6-0$." 7 , based on a spectral energy distribution (SED) simulation, while Podio et al. (2014) derived a 110 au size based on CN 5-4 line observations. T Tau S binary has an extinction of $A_{V}=15$ (Duchêne et al. 2005), much higher than that of $\mathrm{T}$ Tau $\mathrm{N}\left(A_{V}=1.95\right.$, Kenyon \& Hartmann 1995). This difference has been attributed to a compact edge-on circumbinary disk around T Tau S (Duchêne et al. 2005) or blockage of the light due to the circumstellar disk around $\mathrm{T}$ Tau N (Hogerheijde et al. 1997; Beck et al. 2001). Besides the possible edge-on circumbinary disk, $\mathrm{T}$ Tau Sa may be surrounded by an edge-on circumstellar disk of radius 2-3 au (Duchêne et al. 2005) oriented north-south, while the circumstellar disk around $\mathrm{T}$ Tau Sb may not be far from face-on, based on mid-infrared interferometry observations and SED simulations (Ratzka et al. 2009). To date, discussions of the disk structures in this system have mainly been based on indirect measurements. A direct observation of the disks in this system with sufficiently high resolution is crucial for understanding the disk system.

The second issue is the sources of the outflows. Several outflows have been reported in previous observations of this system. Böhm \& Solf (1994) observed T Tau with a spectrograph mounted on the $2.2 \mathrm{~m}$ telescope at the Calar Alto Observatory and reported the discovery of an east-west outflow (hereafter, the E-W outflow) and a southeast-northwest outflow (hereafter, the northwest outflow) from this system. However, it is still under debate which stars trigger which outflow. Some research, e.g., Böhm \& Solf (1994) and
Gustafsson et al. (2010), attributed to T Tau N the E-W outflow and to T Tau $S$ the northwest outflow, but Ratzka et al. (2009) claimed that $\mathrm{T}$ Tau $\mathrm{S}$ is responsible for the E-W outflow. In addition, Gustafsson et al. (2010) suggested the presence of another southwest outflow coming from $\mathrm{T}$ Tau $\mathrm{Sb}$ based on near-infrared hydrogen emission observations. Kasper et al. (2016) also detected a coil-like structure extending to the southwest of the T Tau system in their multi-band near-infrared high-contrast imaging study. This structure can be attributed to a precessing outflow, but it is still unknown whether this originates from $\mathrm{T}$ Tau $\mathrm{N}$ or $\mathrm{S}$. Identification of these outflow features will help determine the evolution of the stars in the multiple system.

Near-infrared high-contrast polarimetry imaging is sensitive to the disk and envelope structures around the young binaries, and can reach a spatial resolution smaller than $0 . " 1$. It has been used to resolve several protoplanetary disks (e.g., Oh et al. 2016). Whitney \& Hartmann (1993) and Murakawa (2010) have simulated the polarized image of YSOs with bipolar outflow cavities, showing that for edge-on disks with outflow cavities, the vectors near the disk plane tend to be aligned with the disk. Therefore, through polarimetry imaging it is possible to detect the outflow cavity structures from the directions of the vectors, and to distinguish the disk and envelope.

In this paper, we will present the results of near-infrared high-contrast polarimetry observations of the T Tau system, and from the results we constrain the disk size around $\mathrm{T}$ Tau $\mathrm{N}$ and S. Section 2 presents the observations and data reduction methods. In Section 3, the observation results are introduced. Section 4 discusses circumstellar disks and outflows based on the observation results. In Section 5, conclusions are made.

\section{Observations and Data Reduction}

Observations of T Tau, part of the Strategic Explorations of Exoplanets and Disks with Subaru survey started in 2009, were conducted on 2015 January 8th, Hawaii Standard Time, using the High Contrast Instrument for the Subaru Next Generation Adaptive Optics (HiCIAO, Tamura et al. 2006) and the adaptive optics (AO) system AO188 (Hayano et al. 2010) mounted on the $8.2 \mathrm{~m}$ Subaru Telescope. The observations were made in the $H$-band, and the quad-polarized differential imaging mode was used in this observation. In this mode, a double-Wollaston prism is used to split the incident light into four $512 \times 512$ pixel channels, corresponding to two o-polarization and two e-polarization channels. The detector has a pixel scale of 9.50 mas pixel $^{-1}$, and the AO system helps to limit the full width at half maximum of the stellar pointspread function to 0. " 07 . To calibrate the Stokes parameters, a half-wave plate was rotated among four angles: $0^{\circ}, 45^{\circ}, 22^{\circ} .5$, and $67^{\circ} .5$. Finally, 36 frames, each with an exposure time of $5 \mathrm{~s}$ and four coadds, were collected, corresponding to a total integration time of 12 minutes.

The data were reduced using the Image Reduction and Analysis Facility pipeline. Generally, the reduction steps include correction of stripes and the flat field, removal of bad pixels and distortions, and generation of a polarized intensity (PI) image. To obtain the PI image, the Stokes $+Q,+U,-Q$, 


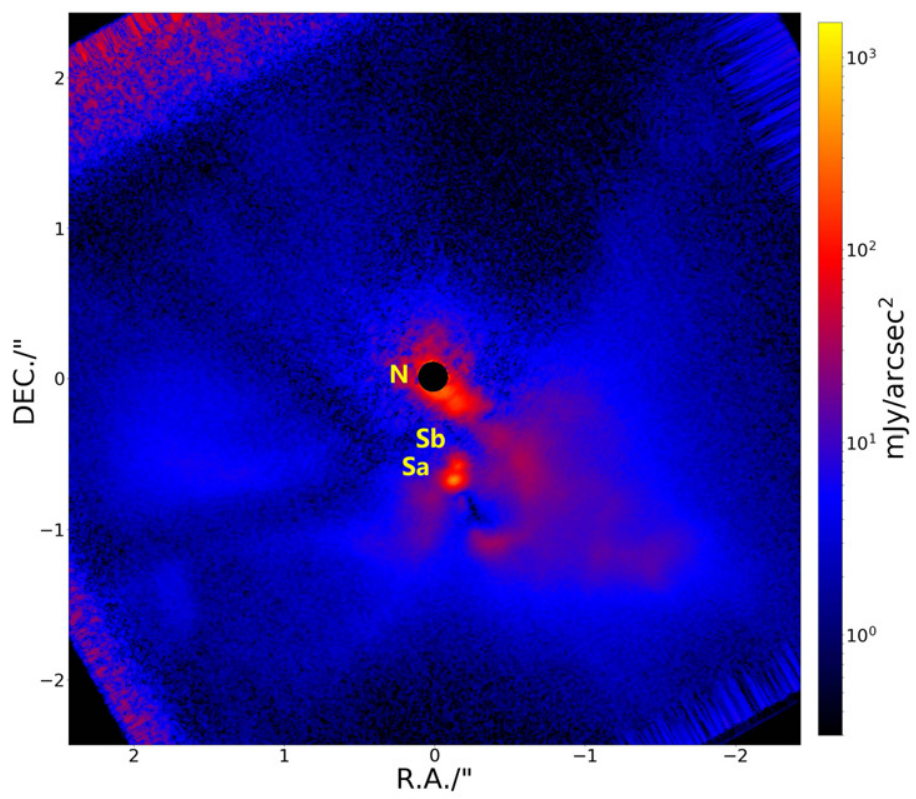

a

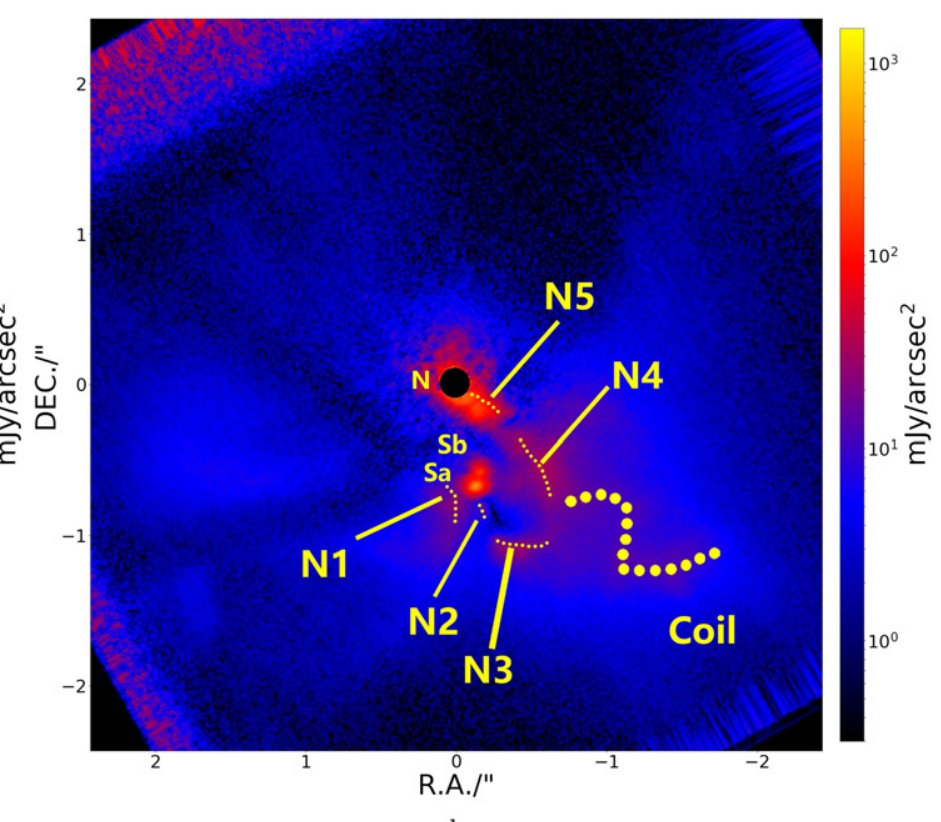

$\mathrm{b}$

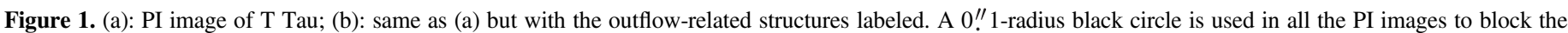
saturated part.

and $-U$ images were first obtained by subtracting the e-images from the o-images, and then the formulas $Q=((+Q)-(-Q) / 2$ and $U=((+U)-(-U)) / 2$ were used to construct the Stokes $Q$ and Stokes $U$ images. After correcting the instrumental polarization, the PI image could be obtained from the Stokes $Q$ and $U$ images using PI $=\sqrt{Q^{2}+U^{2}}$. Through this reduction process, the unpolarized light from the stars is removed, leaving only the polarized light from the circumstellar disk or envelopes. The Stokes I image, or intensity image, which contains both polarized and unpolarized light, was obtained by averaging the sum of the o- and e-images in all frames.

\section{Results}

The PI image of T Tau is presented in Figure 1, showing an area of about 4 !" $9 \times 4$ !. 9 around this triple star system. This is one of the highest-resolution polarization images of $\mathrm{T}$ Tau ever made and has revealed a number of new structures not previously reported. All three stars in this system, T Tau N and $\mathrm{T}$ Tau $\mathrm{Sa} / \mathrm{Sb}$, as well as the nebula-like structures surrounding them, can be seen in this PI image. We measured the distance between T Tau Sa and Sb to be about 0 !" $^{\prime \prime} \pm 0$." 01 , and their position angle to be about $342^{\circ} \pm 1^{\circ}$ (measured from north to east). These results are consistent with those obtained for 2014 December and 2015 January by Köhler et al. (2016), which indicated that the $\mathrm{T}$ Tau $\mathrm{Sa} / \mathrm{Sb}$ system has a separation of 0 "! 11 and a position angle of about $345^{\circ}$ in the $\mathrm{Br} \gamma$ and $\mathrm{Ks}$ bands. $\mathrm{T}$ Tau $\mathrm{N}$ is saturated within about 0 ." 1 , so we use a soft mask to block the saturated part. Even though an accurate determination of the stellar position is not straightforward, the circular shape of the saturation allows us to reasonably estimate its central location within 0 ". 01 . Using the center of the saturated circle as the position of $\mathrm{T}$ Tau $\mathrm{N}$ with a 0 !" 01 uncertainty, the distance between $\mathrm{T}$ Tau $\mathrm{N}$ and $\mathrm{Sa}$ is estimated to be 0 "' $68 \pm 0$ ". 01 , consistent with the result of Köhler et al. (2016) for 2014 December (0."69) in the Br $\gamma$ band. Köhler et al. (2016) determined that the orbit of T Tau N-S has an inclination of about $52^{\circ}$ and a position angle of the ascending node of about $156^{\circ}$. Since the position angle of $\mathrm{T}$ Tau $\mathrm{S}$ relative to $\mathrm{T}$ Tau $\mathrm{N}$ is about $195^{\circ}$ from the PI image, T Tau $\mathrm{S}$ is farther from us than $\mathrm{T}$ Tau N.

Since the light scattered by the dust around the stars is polarized, while the light direct from the stars is non-polarized, polarimetry observations can readily resolve the circumstellar disks and envelopes near the stars. Several petal-like nebular structures that appear to extend from the $\mathrm{T}$ Tau stars are observed, nearly filling the whole field of view.

The polarization vector maps of the system are shown in Figure 2. The vectors show the polarization position angles $\theta_{p}$, which are calculated using the formula $\theta_{p}=0.5 \tan ^{-1}(U / Q)$. It can be seen when the vectors are plotted over these structures that they are centrosymmetric around either $\mathrm{T} \mathrm{Tau} \mathrm{Sa} / \mathrm{Sb}$ or $\mathrm{T}$ Tau N, implying that they are real structures associated with these point sources (e.g., Whitney \& Hartmann 1992). After comparing them with the results of previous observations, these surrounding structures are believed to be the inner part of the envelopes surrounding the triple system, as suggested by Mayama et al. (2006), as well as the circumstellar disk around $\mathrm{T}$ Tau $\mathrm{N}$ and the circumbinary disk around T Tau S. A detailed discussion will be presented in Section 4.1.

The PI image contains certain dark regions, such as a clear large hole in the northwest with an opening angle of about $40^{\circ}$ from $\mathrm{T}$ Tau N. These areas do not exhibit reflected polarized light, but this does not necessarily mean that there is no scattered light or scattering structures (e.g., Perrin et al. 2009). According to Perrin et al. (2009), a fake gap is expected to be caused by backscattered light on the far side of an inclined disk. This is not the case for $\mathrm{T}$ Tau, however, as $\mathrm{T}$ Tau $\mathrm{N}$ has a nearly face-on disk and its surrounding envelopes are not likely to cause backscattered light. Therefore, we suggest that this hole reflects a real structure. In addition, a $J$-band $T$ Tau image obtained by the Canada-France-Hawaii Telescope (Roddier et al. 1999) ${ }^{36}$ also shows a dark region located in the northwest

\footnotetext{
36 A false color image is viewable at http://www.cfht.hawaii.edu/Science/ Astros/Imageofweek/ciw290500.html.
} 

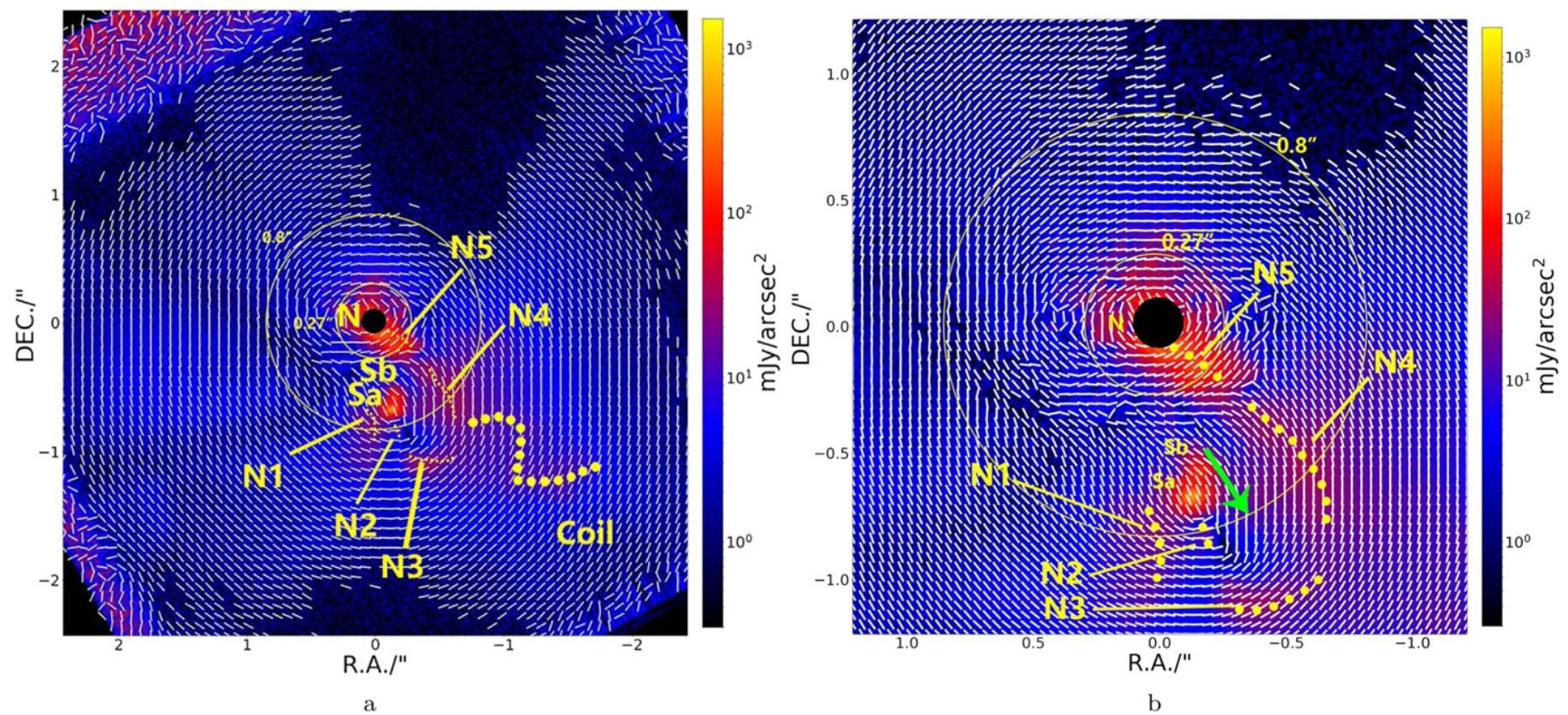

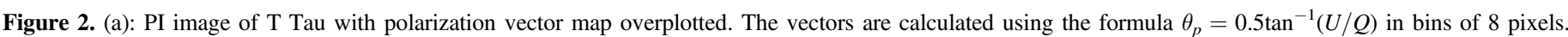

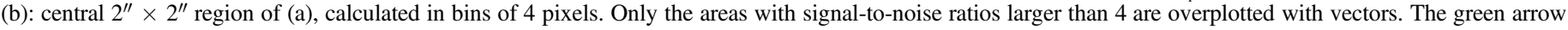
indicates the direction of aligned vectors.

of the nebular structure and its position is near this hole. However, these data are very old so further observations are needed.

In the eastern area, there are two clear dark lines at position angles of $50^{\circ}$ and $100^{\circ}$, which have not been reported in any previous observations. There are also dark regions in the southern and western areas; the south area is about 1"! 8 from T Tau N, while the western one is about 2 "! 2 from T Tau N. In the $H$-band image by Mayama et al. (2006), the southern and western boundaries of the nebular structures detected in the $H$-band are about $2^{\prime \prime}$ from $\mathrm{T}$ Tau $\mathrm{N}$, which is generally consistent with our results. This suggests that these dark regions should represent the boundaries of the envelope.

In the nebular structures, especially in the southwestern area, there are complicated structures, shown in red in the figures. For clarification, they are labeled N1-N5 and "coil" in Figure 1(b). To help understand what these structures are, we made detailed comparisons with previous observations, including the multi-band $(J, H, K$, and several hydrogen emission lines) observations in 2014 and 2015 using VLT/ SPHERE-IRDIS, IRDIFS (Kasper et al. 2016), the $\mathrm{H}_{2} v=$ $1-0 S(1)$ emission line observations performed in 2004 using VLT/SINFONI (Gustafsson et al. 2010), and the $\mathrm{H}_{2} v=$ 1 - OS(1) emission line observations using VLT/NACO with a Fabry-Perot interferometer (Herbst et al. 2007). The comparisons were done by carefully overlapping these images on our image, and matching the positions of the stars (for T Tau $\mathrm{S}$, we use the barycenter as a reference) and scales to see the differences in the structures. A brief summary of the comparison results is given in Table 1.

In our PI image, the N1 structure is associated with T Tau S, and extends to about 0 ". 5 southeast from T Tau Sa. Its position is near the R1 structure reported by Kasper et al. (2016), the 1 and 2 structure reported by Gustafsson et al. (2010), and the C4 structure reported by Herbst et al. (2007), suggesting that they are related.
Table 1

Comparison of Outflow Structures

\begin{tabular}{lccc}
\hline \hline This Paper & $\begin{array}{c}\text { Herbst } \\
\text { et al. (2007) }\end{array}$ & $\begin{array}{c}\text { Gustafsson } \\
\text { et al. (2010) }\end{array}$ & $\begin{array}{c}\text { Kasper } \\
\text { et al. (2016) }\end{array}$ \\
\hline N1 & C4 & $1 \mathrm{a} / \mathrm{b}, 2$ & R1 \\
N2 & $\ldots$ & $\ldots$ & R2 \\
N3 & C3 & 3 & R3 \\
N4 & C1, C2 & 5,6 & R4 \\
\hline
\end{tabular}

Note. This table lists the structures detected in our image and possible related structures in previous observations.

The position of the N2 structure appears to match the position of the R2 structure reported by Kasper et al. (2016), but in our $H$-band image it looks much shorter (about $0 . " 1)$ than the R2 in the $J$-band image of Kasper et al. (2016) (about 0 ."25). The N3 structure is about 0 ." 4 from T Tau Sa and has a length of about 0 ". 4 . Its position also overlaps well with the position of the R3 structure in the $J$-band data of Kasper et al. (2016). It is about $0 !$.'2 below structure 3 reported by Gustafsson et al. (2010) and the C3 structure reported by Herbst et al. (2007).

The N4 structure in our image appears more extended than the R4 structure in Kasper et al. (2016); its brightest part has a length of about $0 . .5$ and width of about 0 !" 2 . This feature covers the positions of the R4 structure reported by Kasper et al. (2016), structures 5 and 6 reported by Gustafsson et al. (2010), the C1 and C2 structures reported by Herbst et al. (2007), and a knot-like structure "N1" reported by Saucedo et al. (2003) in their HST/STIS Ly $\alpha$ observation. Also, it seems to be connected with N3, N5, and the coil in our image.

N5 appears to start at T Tau N and extends to about 0 "! 4 , or $59 \mathrm{au}$, and $\mathrm{PA} \sim 220^{\circ}$ from $\mathrm{T}$ Tau $\mathrm{N}$. This structure does not have any counterparts reported in previous observations and hence, it is a newly detected structure. Although it looks like it is connected with $\mathrm{N} 4$, the polarization vectors plotted over N5 
are centrosymmetric with respect to $\mathrm{T}$ Tau $\mathrm{N}$, while the vectors for $\mathrm{N} 4$ are centrosymmetric with respect to $\mathrm{T}$ Tau $\mathrm{S}$. This implies that N5 and N4 are illuminated by different sources and are therefore different structures. Ray et al. 1997) suggested a structure T Tau R near T Tau N, and Csépány et al. (2015) reported a tentative object 144 mas south of T Tau N. The position of N5 does not fit the object reported by Csépány et al. (2015). If T Tau $R$ is an object orbiting $\mathrm{T}$ Tau $\mathrm{N}$, its position angle should have changed from about $44^{\circ}$ to about $240^{\circ}$ over the past 22 years, considering its location to be $0 . " 3$, or $44 \mathrm{au}$, from T Tau N. As a result, N5 is also unlikely to be related to $\mathrm{T}$ Tau R. In addition, the vectors plotted over N5 surround only $\mathrm{T}$ Tau N, thus another luminous object near it can be excluded. Therefore, we conclude that N5 could be part of the circumstellar disk around $\mathrm{T}$ Tau $\mathrm{N}$.

A coil structure, first reported by Kasper et al. (2016), is clearly seen in our PI image. Its appearance is similar to the $J$-band structure observed by Kasper et al. (2016). In our image, it looks like it can be connected with the N4 structure, and extends to about 2." 1 , or 294 au, with a position angle of about $230^{\circ}$ from T Tau N. The T Tau NW structure, which was reported by Herbst et al. (2007) and could be related to the northwest outflow, is not found in our image. After comparing with the results of Kasper et al. (2016), it is likely that this structure is just at the northwestern edge of our field of view and cannot be seen in our image.

\section{Discussion}

\subsection{Circumstellar Disks}

In this section, we focus on the circumstellar disks in the $\mathrm{T}$ Tau system. Akeson et al. (1998) reported that T Tau N might have a nearly face-on disk with an outer radius of about $41 \mathrm{au}$, and previous SED fitting (Gustafsson et al. 2008) and CN line observations (e.g., Podio et al. 2014) have suggested that it has a circumstellar disk of about 100 au. A disk of this scale should be detectable within the field view of our observation, although it is not easy to distinguish from the envelope. The structures around T Tau N close to 0"! 11 (16 au) may correspond to the previously reported circumstellar disks.

The circumstellar disk of $\mathrm{T}$ Tau $\mathrm{N}$ should not exceed the inner boundary of the "hole" to the northwest of T Tau $\mathrm{N}$ of about 0 " 8 , or 117 au. However, in the south, the vector map in Figure 2 shows that only the vectors with distances smaller than $\sim 0$.'27, or $40 \mathrm{au}$, are centrosymmetric with respect to $\mathrm{T}$ Tau N. As described in Section 3, N4 and N5 should be considered independent structures, and structure N5 is likely to be related to $\mathrm{T}$ Tau $\mathrm{N}$, because the vectors on it are centrosymmetric with respect to T Tau N. Even if N5 is part of the disk, its outer size will be only about $59 \mathrm{au}$.

It would be strange for the disk to be so asymmetric. In addition, Miranda \& Lai (2015) showed that for an equal-mass binary, the circumstellar disk size decreases as the orbital eccentricity increases. The mass ratio $q\left(q=M_{b} /\left(M_{a}+M_{b}\right)\right.$, where $M_{a}$ and $M_{b}$ represent the masses of the binary primary star and secondary star, respectively) of the T Tau N-S system is about 0.43 , not far from 0.5 , and based on the best orbital parameters derived by Köhler et al. (2016), namely that the $\mathrm{N}-\mathrm{S}$ orbit has a semimajor axis of about $430 \mathrm{au}$ and an eccentricity about 0.7 , the circumstellar disk size should be at least smaller than 0.2 times the orbital semimajor axis, i.e., $86 \mathrm{au}$. In this case, a disk with a radius of about $117 \mathrm{au}$ is obviously too large. It is possible that either the boundary of the envelope is at $117 \mathrm{au}$, i.e., the actual radius of the disk is much smaller than $117 \mathrm{au}$, or the $\mathrm{N}-\mathrm{S}$ orbit parameters derived by Köhler et al. (2016) are not accurate (e.g., the semimajor axis of the N-S orbit derived by Köhler et al. 2016 ranges from 180 to $1220 \mathrm{au}$ ) and the real orbit may have a larger semimajor axis and smaller eccentricity. At any rate, based on our observation results, the disk in the south of $\mathrm{T}$ Tau $\mathrm{N}$ extends to no more than 0.27 , or $40 \mathrm{au}$, while in the northwest, its size should be smaller than 0 ". 8 , or 117 au.

For T Tau S, some vectors, such as those to the northeast and southwest of the stars, appear to be aligned in one direction, with position angles of about $30^{\circ} / 210^{\circ}$, as indicated by the green arrow in Figure 2(b), while the vectors to the southeast and northwest of the stars show centrosymmetric characteristics, and they still have a tendency to be aligned with a position angle of about $30^{\circ} / 210^{\circ}$. This "aligned" centrosymmetry is consistent with the presence of a "polarization disk" (e.g., Tamura et al. 1991; Whitney \& Hartmann 1993; Murakawa 2010) and is considered to result from multiple scattering in dense regions of the disk. The light is scattered multiple times in an optically thick disk, but is scattered less in an optically thin region, like bipolar outflow cavities. According to the simulation results of Murakawa (2010), the vectors near the disk plane seem to be aligned with the disk's orientation, but the vectors in other areas, such as the outflow cavities, will still be centrosymmetric, as the light in those regions may only be scattered once. Considering that $\mathrm{T}$ Tau $\mathrm{S}$ itself is a class I object, this is a reasonable explanation. Also, an optically thick inclined disk will cause a large extinction, which is consistent with previous findings that $\mathrm{T}$ Tau $\mathrm{S}$ has a very large extinction. The rough range of the "polarization disk" is consistent with the real disk size, as suggested by Murakawa (2010), and based on this we can estimate that the disk should have a radius of about 0.13 , or $44 \mathrm{au}$, with a position angle of about $30^{\circ}$. The actual inclination of this circumbinary disk is hard to estimate from just the vector map, while in the simulation of Murakawa (2010), the aligned vectors near the disk plane appeared for $H=0.3$ and $\theta_{\text {inc }}=60^{\circ}$ and with a small maximum dust size $\alpha_{\max }=$ $0.25 \mu \mathrm{m}$. Considering that the actual maximum dust size in the disk around $\mathrm{T}$ Tau $\mathrm{S}$ could be larger, its inclination should also be considered to be larger, to align the vectors with the disk. Therefore, it seems that the inclination of the disk should be larger than $60^{\circ}$. We hope future ALMA observations of the gas velocity around the disk will lead to a more accurate result.

Binaries will open central gaps in circumbinary disks, and for circumbinary disks that are coplanar with the binary orbital planes, the gap size is usually about 2-3 times the binary semimajor axis (e.g., Artymowicz \& Lubow 1994). For a circumbinary disk that is misaligned with the binary orbital plane, the opened gap size seems to be smaller (e.g., Miranda \& Lai 2015). Miranda \& Lai (2015) calculated the gap size of circumbinary disks under different misaligned angles relative to the binary orbit and eccentricities (Figure 9 of Miranda \& Lai 2015) around binaries with mass ratios of $q=0.1,0.3$, and 0.5 . Considering that the mass ratio of $\mathrm{T}$ Tau $\mathrm{S}$ is about 0.2 , the opened gap size can be estimated from the $q=0.1$ and 0.3 cases. The orbit of $\mathrm{T}$ Tau $\mathrm{S}$ has a semimajor axis of about $13 \mathrm{au}$, an eccentricity of about 0.56 , and an inclination of about $20^{\circ}$. If the circumbinary disk around $\mathrm{T}$ Tau $\mathrm{S}$ has an inclination 


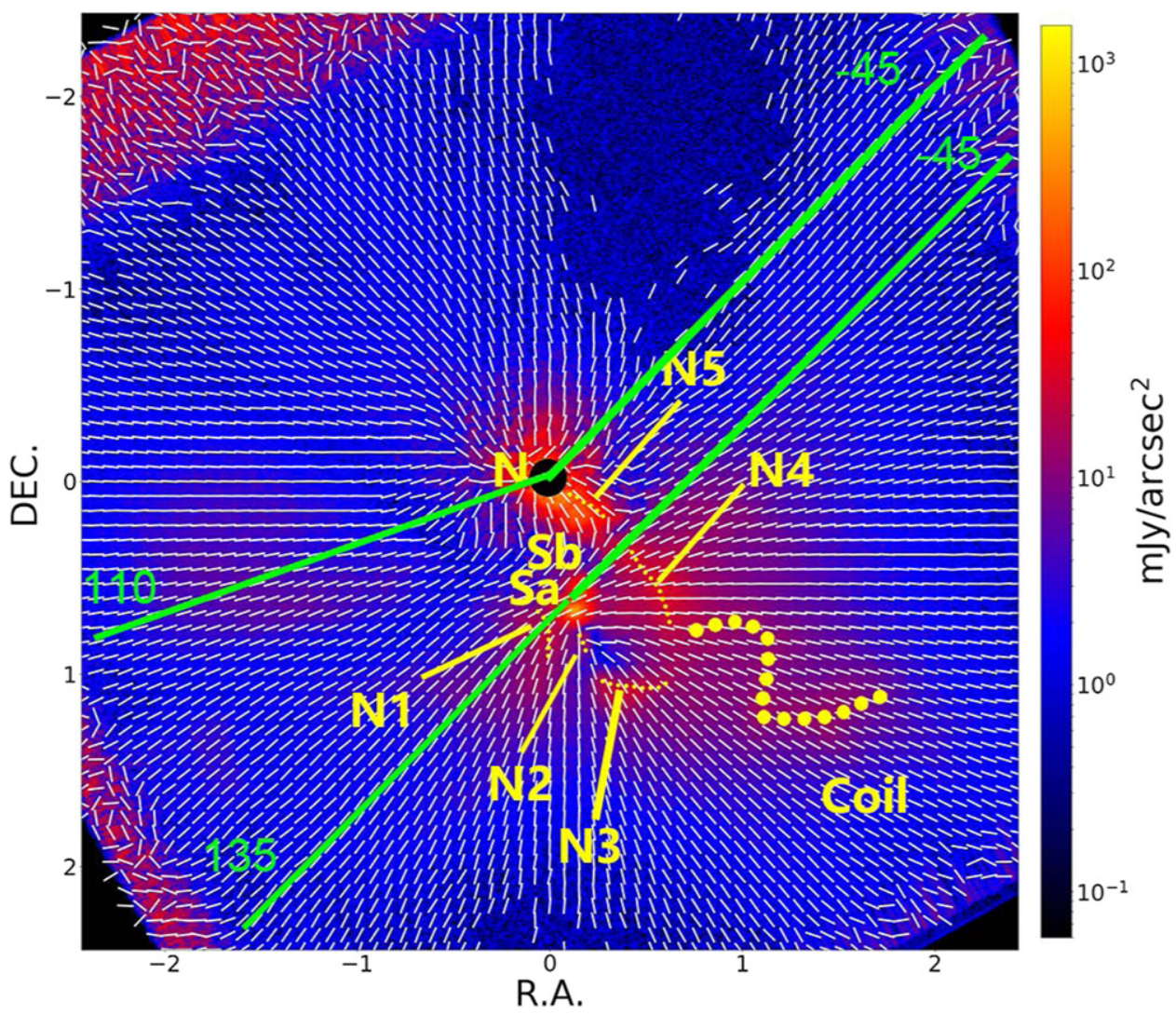

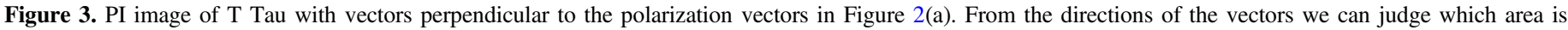

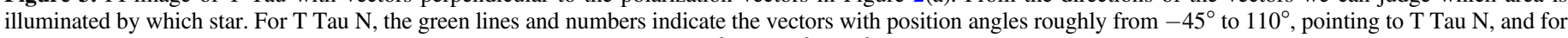
$\mathrm{T}$ Tau $\mathrm{S}$ they represent the vectors with position angles roughly from $135^{\circ}$ to $-45^{\circ}\left(315^{\circ}\right)$.

larger than about $60^{\circ}$ as we suggested, the misaligned angle between the disk and orbital plane is larger than about $40^{\circ}$. Figure 9 of Miranda \& Lai (2015) shows that for a binary mass ratio of $q=0.1$ and 0.3 , orbital eccentricity of 0.56 and disk misaligned angle of $45^{\circ}$, the opened gap size is about 2.5 times the binary semimajor axis, so it is reasonable to suggest that for $q=0.2$ and misaligned angle $40^{\circ}$, the opened gap size should be close to 2.5 times the binary semimajor axis. Miranda \& Lai (2015) showed that the larger the inclination between the binary orbit and disk, the smaller the gap size that the binary opens. Therefore, the gap in the circumbinary disk of $\mathrm{T}$ Tau $\mathrm{S}$ should be smaller than 32.5 au.

\subsection{Outflows}

In this section, we briefly analyze the structures associated with the outflows. To help in this analysis, we briefly discuss the polarization vectors and the relative distance of $\mathrm{T}$ Tau $\mathrm{N}$ and $\mathrm{S}$.

In general, the polarization vectors on real structures, e.g., circumstellar disks and envelopes, will be centrosymmetric around the central star. If we draw perpendicular vectors relative to the polarization vectors, then we will see that the vectors point to the central star. In binary/multiple star systems, this can help us judge which star illuminates the structures. This image is presented in Figure 3. From this image, we can see that for $\mathrm{T}$ Tau $\mathrm{N}$ the vectors with position angles from roughly $-45^{\circ}$ to $110^{\circ}$ relative to $\mathrm{T}$ Tau $\mathrm{N}$ point to $\mathrm{T}$ Tau N , indicating that they are illuminated by T Tau N. For T Tau $S$, the vectors with position angles from roughly $135^{\circ}$ to $-45^{\circ}$ point to $\mathrm{T}$ Tau $\mathrm{S}$, indicating that they are illuminated by $\mathrm{T}$
Table 2

Summary of Outflows

\begin{tabular}{lcc}
\hline \hline Star & Structure & Outflow \\
\hline T Tau S & N1, N4 & E-W \\
T Tau S & N2, N3 & South \\
T Tau S & Coil & Southwest \\
T Tau N & e.g., Herbst et al. (2007) & Northwest
\end{tabular}

Tau S. For the other areas of the envelopes, most of the vectors appear to point to the area between $\mathrm{T}$ Tau $\mathrm{N}$ and $\mathrm{T}$ Tau $\mathrm{S}$, indicating that they are illuminated by both stars. Considering that $\mathrm{T}$ Tau $\mathrm{S}$ is located generally south (position angle $\sim 195^{\circ}$ ) of $\mathrm{T}$ Tau N, we expect that the areas illuminated by T Tau N and $\mathrm{S}$ are generally horizontal symmetric in Figure 3. However, in Figure 3, they are generally symmetric, with position angles of about $-45^{\circ}$. This may indicate some internal structures in the envelope, but further observations are necessary to confirm it.

As mentioned in Section 4.1, N5 appears to be part of the circumstellar disk around T Tau N. The other structures, N1-N4, as well as the coil, could be related to the outflows in this system. The T Tau S system, as discussed in Section 4.1, has a nearly edge-on disk oriented at about $30^{\circ}$, and $\mathrm{N} 1$ and $\mathrm{N} 4$ are located at its opposite sides. Therefore, we suggest that $\mathrm{N} 1$ and N4 could represent two opposite outflow cavities of T Tau S.

Based on the simulation results reported by Murakawa (2010), the direction of the aligned vectors should be generally perpendicular to the direction of the bipolar outflow cavity. Therefore, from the vector map, we can estimate the position 


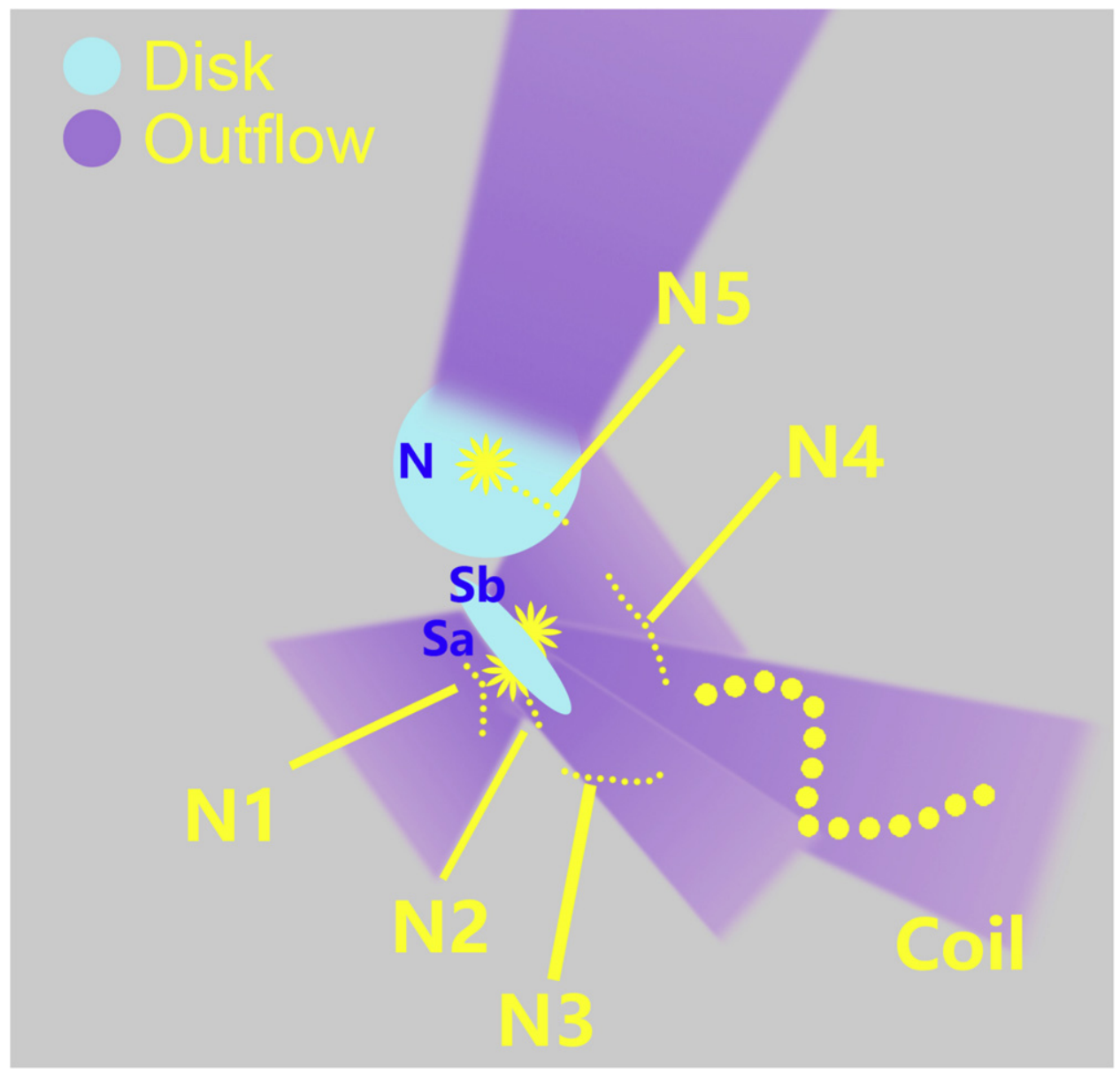

Figure 4. Illustration of suggested $\mathrm{T}$ Tau surrounding structures. The three stars are believed to be surrounded by envelopes (gray background). There are several possible outflows from this system (purple). In addition, both the T Tau N and T Tau S systems have disks (blue).

angles of the outflow cavities to be about $120^{\circ}$ and $300^{\circ}$. Eislöffel \& Mundt (1998) derived the tangential velocity position angle for $\mathrm{HH} 155$, an object located $\sim 30^{\prime \prime}$ west of T Tau and believed to be caused by the E-W outflow, to be about $305^{\circ}$, near the position angle we derived. Also, Kasper et al. (2016) noted a T Tau SE structure in their $\mathrm{H}_{2}$ image, even though the signal-to-noise ratio was too low to confirm its presence. We note that its position angle is $119^{\circ}$, which is close to the position angle of the outflow cavities we derived. We suggest that this T Tau SE could be a real structure related to this near eastern-western outflow. In summary, we suggest that the well-known E-W outflow is triggered by T Tau S and N1 and $\mathrm{N} 4$ represent the bipolar outflow cavities related to this outflow.

For the other structures, Gustafsson et al. (2010) suggested that their structure 3 could represent a southwest outflow, which we here refer to as a "south" outflow, to distinguish from the southwest outflow corresponding to the coil. Since our N2 and N3 structures are located near their structure 3, their connection to the outflow is readily implied. The coil, as Kasper et al. (2016) suggested, is likely to be a precessing outflow, but its origin remains a mystery. There have been no previous reports on this structure in Kasper et al. (2016). A possible related report is that of a "bow" structure by Gustafsson et al. (2010).

The average position angle of this coil is about $249^{\circ}$ $\left(\sim 237^{\circ}-261^{\circ}\right)$ relative to $\mathrm{T}$ Tau $\mathrm{S}$ and $229^{\circ}\left(\sim 221^{\circ}-237^{\circ}\right)$ relative to T Tau N. The vectors in Figure 3 on the coil point toward T Tau S, with an average position angle of about $250^{\circ}$ (corresponding to an average polarization position angle of about $160^{\circ}$ ) rather than $\mathrm{T}$ Tau $\mathrm{N}$, indicating that the coil structure is illuminated by T Tau S. Since T Tau N is in front of $\mathrm{T}$ Tau $\mathrm{S}$, if the coil is one of the outflow structures emitted by $\mathrm{T}$ Tau N, we expect to see the vectors in Figure 3 pointing to $\mathrm{T}$ Tau $\mathrm{N}$ rather than $\mathrm{T}$ Tau $\mathrm{S}$. Thus, we suggest that the coil is more likely to be related to the T Tau $\mathrm{S}$ system. In this case, there are three outflows that could be related to $\mathrm{T}$ Tau S, though only two stars have so far been detected in this system. A simple hypothesis is that there is another companion star that has not yet been detected. Another possibility is that some structures, such as N2 and N3, are not caused by an outflow but by an inflow. Future observations, especially high-resolution spectroscopy observations and high-resolution $\mathrm{CO}$ gas emission line observations like ALMA, will help resolve this problem.

In addition, the T Tau NW structure is possibly located at the northwest edge of the field of view near the big hole, so we suggest that this hole is likely to be caused by the northwest outflow. Since the vectors in Figure 3 of the envelope structures near the hole generally point to $\mathrm{T}$ Tau $\mathrm{N}$, it is likely that the remaining northwest outflow is related to $\mathrm{T}$ Tau $\mathrm{N}$.

A summary of our interpretation regarding the outflows is listed in Table 2 and illustrated in Figure 4. There have been no previous reports on the relative distances of the outflow-related structures N1-N5 and the coil. In our interpretations, N1-N4 and the coil should be near T Tau S, i.e., farther than T Tau N and N5. Knowing the velocities of these structures may help determine more accurate relative distances. In addition, just 
from near-infrared polarimetry observations it is hard to judge which outflows are actually triggered by $\mathrm{T}$ Tau Sa or T Tau Sb. We hope ALMA observations can provide further information regarding this issue.

\section{Conclusion}

Using the HiCIAO instrument mounted on the Subaru Telescope, we resolved the nearby structures around the T Tau triple star system and derived new insights into the disks and outflows in this system. From the PI image we found that this triple system is surrounded by petal-like envelopes, and some structures in these envelopes could be associated with outflows from the stars. Considering the existence of a hole in the northwest, we suggest that the face-on disk of $\mathrm{T}$ Tau $\mathrm{N}$ should not exceed about 0 ". 8 , or $117 \mathrm{au}$, in this direction, and it is no more than about 0.27 , or $40 \mathrm{au}$, in the south. Also, we discovered a new structure N5, extending to about 59 au from $\mathrm{T}$ Tau N, which could be part of the disk around T Tau N. For T Tau $\mathrm{S}$, the vector map implies that it has a highly inclined disk with a radius of about 44 au, with a position angle of about $30^{\circ}$.

We also investigated the sources of previously discovered outflows. We believe that $\mathrm{T}$ Tau $\mathrm{S}$ triggers the well-known E-W outflow, because it has a nearly north-south circumbinary disk, and the outflow-related structures N1 and N4 are located opposite this outflow. Since the vectors on the coil in Figure 3 point to $\mathrm{T}$ Tau $\mathrm{S}$ rather than $\mathrm{T}$ Tau $\mathrm{N}$, we conclude that the coil is located in the envelope around $\mathrm{T}$ Tau $\mathrm{S}$ and hence is more likely to be triggered by T Tau S than T Tau N. The N2 and N3 structures appear to represent an outflow extending to the south, though if this is the case there should be one more star, currently undetected, in the T Tau S system. Finally, we have detected a large hole to the northwest of $\mathrm{T}$ Tau $\mathrm{N}$ and the vectors in Figure 3 point to $\mathrm{T}$ Tau $\mathrm{N}$, it is possible that the Northwest outflow is related to this hole.

We thank the anonymous reviewer, who has greatly helped us improve this paper. This study was based on data collected by the Subaru Telescope, which is operated by the National Astronomical Observatory of Japan (NAOJ) and National Institutes of Natural Sciences (NINS). We thank the Subaru Telescope staff for their support during the observations. We also acknowledge the access given to us of the SIMBAD database operated by the Strasbourg Astronomical Data Center (CDS), Strasbourg, France. IRAF is distributed by the National Optical Astronomy Observatory, which is operated by the Association of Universities for Research in Astronomy (AURA) under a cooperative agreement with the National Science Foundation. M.T. is supported by a Grant-in-Aid for Scientific Research (No.15H02063). J.C. is supported by the U.S. National Science Foundation under Award No. 1009203. The authors wish to recognize and acknowledge the very significant cultural role and reverence that the summit of Maunakea has always had within the indigenous Hawaiian community. We are most fortunate to have the opportunity to conduct observations from this mountain.

\section{ORCID iDs}

Yi Yang (D) https://orcid.org/0000-0002-9024-4150 Satoshi Mayama (1) https://orcid.org/0000-0002-3424-6266 Eiji Akiyama (i) https://orcid.org/0000-0002-5082-8880 Thayne Currie (ib https://orcid.org/0000-0002-7405-3119
Markus Janson (ib https://orcid.org/0000-0001-8345-593X Munetake Momose (iD https://orcid.org/0000-0002-3001-0897 Takao Nakagawa (iD https://orcid.org/0000-0002-6660-9375 Daehyeon Oh (1) https://orcid.org/0000-0003-2691-804X Tomoyuki Kudo (iD https://orcid.org/0000-0002-9294-1793 Timothy D. Brandt (i) https://orcid.org/0000-0003-2630-8073 Olivier Guyon (ib https://orcid.org/0000-0002-1097-9908 Yutaka Hayano (iD https://orcid.org/0000-0003-4937-4233 Klaus W. Hodapp (iD https://orcid.org/0000-0003-0786-2140 Ryo Kandori (iD https://orcid.org/0000-0003-2610-6367 Gillian R. Knapp (i) https://orcid.org/0000-0002-9259-1164 Jungmi Kwon (iD https://orcid.org/0000-0003-2815-7774 Shoken Miyama (1) https://orcid.org/0000-0001-5017-180X Amaya Moro-martin (iD https://orcid.org/0000-00019504-8426

Tae-Soo Pyo (iD https://orcid.org/0000-0002-3273-0804 Michihiro Takami (1) https://orcid.org/0000-0001-9248-7546 Hiroshi Terada (i) https://orcid.org/0000-0002-7914-6779 Christian Thalmann (iD https://orcid.org/0000-0002-1664-2177 Makoto Watanabe (iD https://orcid.org/0000-0002-3656-4081 John Wisniewski (i) https://orcid.org/0000-0001-9209-1808 Tomonori Usuda (iD https://orcid.org/0000-0001-9855-0163 Motohide Tamura (iD https://orcid.org/0000-0002-6510-0681

\section{References}

Akeson, R. L., Koerner, D. W., \& Jensen, E. L. N. 1998, ApJ, 505, 358 Artymowicz, P., \& Lubow, S. H. 1994, ApJ, 421, 651

Beck, T. L., Prato, L., \& Simon, M. 2001, ApJ, 551, 1031

Böhm, K.-H., \& Solf, J. 1994, ApJ, 430, 277

Csépány, G., van den Ancker, M., Ábrahám, P., Brandner, W., \& Hormuth, F. 2015, A\&A, 578, L9

Duchêne, G., Ghez, A. M., McCabe, C., \& Ceccarelli, C. 2005, ApJ, 628, 832 Dyck, H. M., Simon, T., \& Zuckerman, B. 1982, ApJL, 255, L103

Eislöffel, J., \& Mundt, R. 1998, AJ, 115, 1554

Furlan, E., Hartmann, L., Calvet, N., et al. 2006, ApJS, 165, 568

Gustafsson, M., Kristensen, L. E., Kasper, M., \& Herbst, T. M. 2010, A\&A, 517, A19

Gustafsson, M., Labadie, L., Herbst, T. M., \& Kasper, M. 2008, A\&A, 488,235

Hayano, Y., Takami, H., Oya, S., et al. 2010, Proc. SPIE, 7736, 77360N

Herbst, T. M., Hartung, M., Kasper, M. E., Leinert, C., \& Ratzka, T. 2007, AJ, 134,359

Hogerheijde, M. R., van Langevelde, H. J., Mundy, L. G., Blake, G. A., \& van Dishoeck, E. F. 1997, ApJL, 490, L99

Kasper, M., Santhakumari, K. K. R., Herbst, T. M., \& Köhler, R. 2016, A\&A, 593, A50

Kenyon, S. J., \& Hartmann, L. 1995, ApJS, 101, 117

Köhler, R., Kasper, M., Herbst, T. M., Ratzka, T., \& Bertrang, G. H.-M. 2016, A\&A, 587, A35

Koresko, C. D. 2000, ApJL, 531, L147

Loinard, L., Torres, R. M., Mioduszewski, A. J., et al. 2007, ApJ, 671, 546

Luhman, K. L., Allen, P. R., Espaillat, C., Hartmann, L., \& Calvet, N. 2010, ApJS, 186, 111

Mayama, S., Tamura, M., Hayashi, M., et al. 2006, PASJ, 58, 375

Miranda, R., \& Lai, D. 2015, MNRAS, 452, 2396

Murakawa, K. 2010, A\&A, 518, A63

Oh, D., Hashimoto, J., Carson, J. C., et al. 2016, ApJL, 831, L7

Perrin, M. D., Schneider, G., Duchene, G., et al. 2009, ApJL, 707, L132

Podio, L., Kamp, I., Codella, C., et al. 2014, ApJL, 783, L26

Ratzka, T., Schegerer, A. A., Leinert, C., et al. 2009, A\&A, 502, 623

Ray, T. P., Muxlow, T. W. B., Axon, D. J., et al. 1997, Natur, 385, 415

Roddier, C., Roddier, F., Graves, J. E., et al. 1999, in ESO Conf. and Workshop Proc. 56, Astronomy with Adaptive Optics: Present Results and Future Programs, ed. D. Bonaccini (Garching: ESO), 389

Saucedo, J., Calvet, N., Hartmann, L., \& Raymond, J. 2003, ApJ, 591, 275

Tamura, M., Gatley, I., Joyce, R. R., et al. 1991, ApJ, 378, 611

Tamura, M., Hodapp, K., Takami, H., et al. 2006, Proc. SPIE, 6269, 62690V

Whitney, B. A., \& Hartmann, L. 1992, ApJ, 395, 529

Whitney, B. A., \& Hartmann, L. 1993, ApJ, 402, 605 